\title{
The Financial Effect of Medicare Coverage Design and Safety Net Options for Cancer Care
}

Stephanie S. Bethay, PharmD, MBA, BCPS; Matthew J. Travis, PharmD; Stephen K. Batt, PharmD, CSP; Samantha H. Bochenek, PharmD, MBA, BCPS; and Philip A. Schwieterman, PharmD, MHA

\begin{abstract}
BACKGROUND: National spending on specialty medications accounted for approximately $\$ 193$ billion in 2016. The coverage design for Medicare Parts B and D has shifted medication costs to patients, which may prohibit patients from starting or maintaining therapy due to affordability. As a result, patients have enrolled in safety net financial options, such as patient assistance and foundation programs. Safety net options may provide savings not otherwise realized by Medicare; however, they may have a negative financial effect on health systems and pharmaceutical manufacturers.
\end{abstract}

OBJECTIVES: To (a) quantify financial savings to Medicare as a result of patient enrollment in patient assistance programs and (b) quantify the financial effect of safety net options for patients, manufacturers, and the academic medical center that participated in this study.

METHODS: A single-center, nonrandomized, retrospective pilot study of Medicare beneficiaries was conducted. Patients who were prescribed hematology/oncology specialty medications and enrolled in safety net options between July 2015 and June 2017 were included. Investigators collected data related to fill history, drug cost, and prescription coverage. The primary outcome was the overall cost savings to Medicare as a result of patient enrollment in patient assistance programs. Secondary outcomes included total patient out-of-pocket savings as a result of foundation copayment support, financial effect on manufacturers as a result of patient assistance programs, and health system revenue impact as a result of safety net options. Descriptive statistics were used.

RESULTS: This study included 114 patients. Medicare saved $\$ 5,083,816.83$ over 2 years as a result of patient assistance programs. Eight foundations provided $\$ 240,350.04$ in patient insurance copayments. Nine manufacturers provided 2,243 free drug doses, valued at $\$ 3,379,032.34$. The participating medical center missed the opportunity for $\$ 6,481,543.55$ in revenue due to patient assistance programs.

CONCLUSIONS: The participating medical center's efforts to improve access to oncology care took considerable time and resources. These activities, as well as unreimbursed infusion services, were costs to the medical center that may not be recognized by Medicare. Manufacturers also supported patient access through their sponsored patient assistance programs. The use of these services and safety net options resulted in cost savings to Medicare and their beneficiaries.

J Manag Care Spec Pharm. 2020;26(1):76-80

Copyright $\odot 2020$, Academy of Managed Care Pharmacy. All rights reserved.

\section{What is already known about this subject}

Specialty medications often are associated with elevated out-ofpocket costs, which may affect patient adherence to therapy.

Safety net options have been shown to increase patient access to medications not otherwise fully covered by insurance.

\section{What this study adds}

In this study, patient assistance programs were associated with savings to Medicare that may not be recognized because of the lack of claim payments.

Safety net options were associated with savings in patient insurance copayments.

Health care providers dedicate resources to enroll patients in safety net options and ensure access to medications.

A ccording to Medicare, specialty medications are selfadministered and professionally administered medications that cost $\$ 670$ or more per month. ${ }^{1}$ From a Medicare standpoint, these drugs are generally covered under Medicare Part B (professionally administered) or Medicare Part D (self-administered). ${ }^{2,3}$ Specialty medications accounted for $42.9 \%$ of all drug costs in 2016, with an estimated cost of $\$ 193$ billion. ${ }^{4}$ In 2017 , specialty medications accounted for approximately $46.5 \%$, or $\$ 407$, of $\$ 876$ net dollars per person per year spent on medications. These medications drove $\$ 9.8$ billion of $\$ 12$ billion net growth of new brands in 2017. ${ }^{5}$

Medicare is the largest payer of prescription drug coverage nationally. ${ }^{6}$ Copayments are often elevated for specialty medications, which shifts cost burden significantly to patients. ${ }^{3}$ One study estimated that some Medicare beneficiaries using cancer medications long term will spend approximately $\$ 4,000$ $\$ 10,000$ out of pocket per year. ${ }^{7}$ Because of high out-of-pocket costs and prior authorization requirements, patients may abandon or delay therapy. ${ }^{8}$ Medicare plans primarily cover indications approved by the U.S. Food and Drug Administration, and drugs that are approved under the Compendia used by the Medicare program may not cover all disease states. When therapy is medically necessary, it still may not be covered. Medicare patients also do not qualify for manufacturer-sponsored copayment cards that help to make medications more 


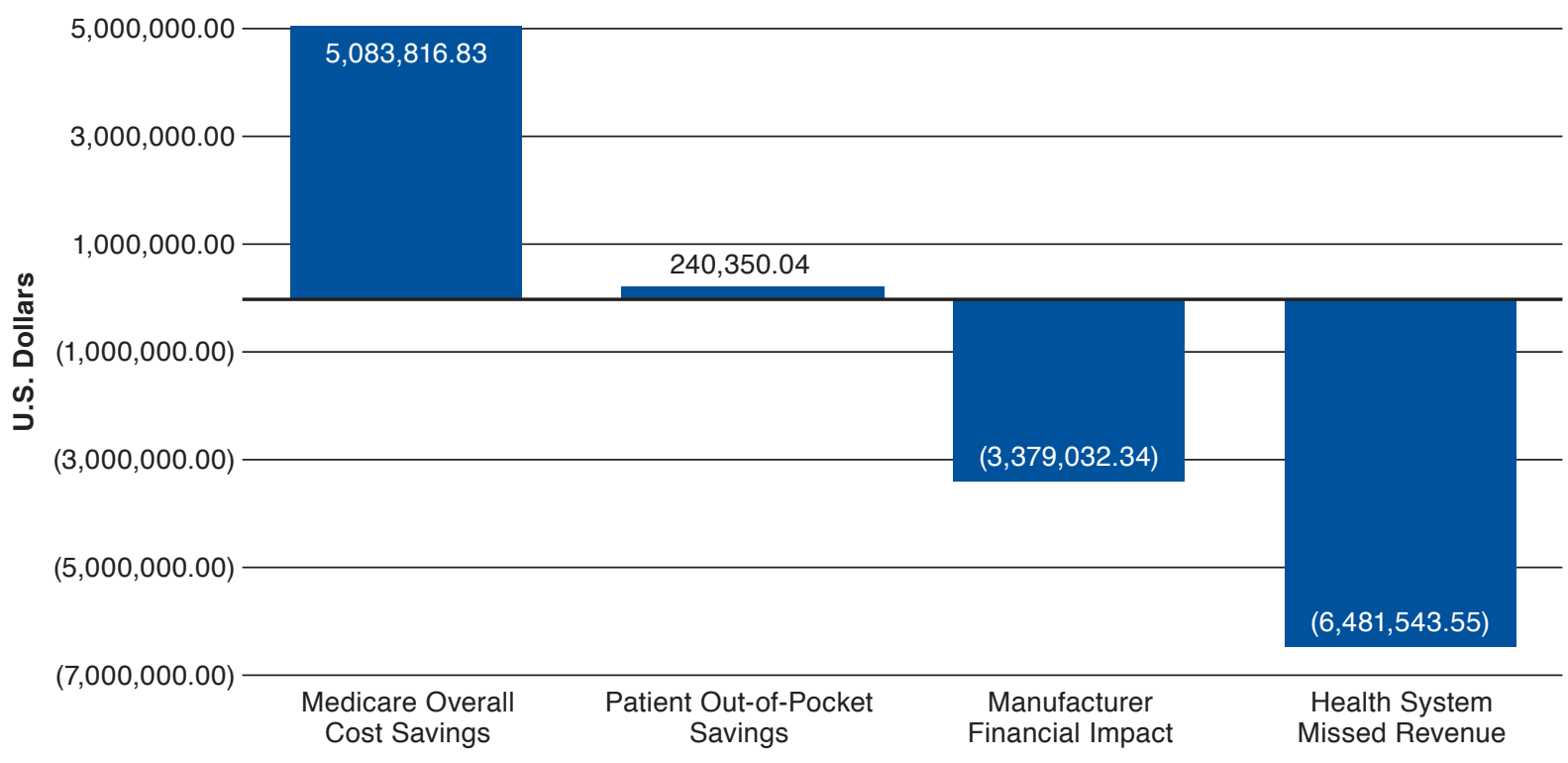

affordable. As a result of these challenges, patients enroll in safety net options, such as manufacturer-free drugs (referred to here as "patient assistance programs") and foundation copayment support programs. ${ }^{3,9}$

Using these safety net options subsequently decreases claim submissions to Medicare. Manufacturers are also directly affected by the safety net options in which Medicare beneficiaries are enrolled. Secondary to unaffordable copayments, many manufacturers offer patient assistance programs to increase patient access to expensive medications that patients may not otherwise receive. ${ }^{10}$ Finally, health systems are affected by patient enrollment in safety net options. Health systems must dedicate labor resources to manage patient enrollment in safety net options and inventory management of medications received through patient assistance programs. Also, health systems do not receive Medicare reimbursement for dispensing medications obtained through patient assistance programs. If safety net options are not pursued, however, patients may be asked to pay expenses out of pocket, or health care providers can choose not to service the patient. This alternative is less than ideal, since it does not support the mission of safety net health care institutions.

The purpose of this study was to (a) quantify the financial effect of Medicare coverage design on patients, manufacturers, and health care institutions and (b) quantify the total cost savings to Medicare as a result of patient use and participation by manufacturers and health care institutions in safety net options.

\section{Methods}

The study design was a single-center, nonrandomized, retrospective pilot study of hematology/oncology patients at a large academic medical center from July 1, 2015, to June 30, 2017 (fiscal years 2016-2017). Inclusion criteria included Medicare beneficiaries with a malignant hematology or oncology diagnosis who received specialty medications dispensed by the in-house specialty pharmacy or infusion center. Patients were only included in the study if they were enrolled in a safety net option through a patient assistance program or health care foundation. All included patients received specialty hematology/oncology medications on an outpatient basis. Oral specialty medications were dispensed and delivered through a health system specialty pharmacy, and injectable specialty medications were administered within a hospital-based, outpatient infusion center. While the Medicare specialty definition is now medications that cost at least $\$ 670$ monthly, the period of this study was primarily before the application of this new definition, thus, $\$ 600$ was used as the price point for the inclusion of specialty medications. ${ }^{3}$

Patients were identified by a query of the electronic health record, the specialty pharmacy dispensing system, and the specialty pharmacy clinical management system. Patient demographic information and specialty medication dispensing data were collected. Manufacturers were contacted to collect patient fill history for drugs furnished at no cost by patient assistance programs. This study was approved by the Institutional Review Board of the University of Kentucky. Encrypted campus 


\section{TABLE 1 Calculations for Financial Effect of Patient Enrollment in Safety Net Options}

\begin{tabular}{|c|c|c|}
\hline $\begin{array}{l}\text { Medicare overall } \\
\text { cost savings }\end{array}$ & $\$ 5,083,816.83$ & $\begin{array}{l}\text { Sum of reimbursement savings = total reimbursement savings for Part B PAP drugs + total reimbursement savings } \\
\text { for Part D PAP drugs } \\
\text { Explanation of reimbursement savings calculations: } \\
\text { Total reimbursement savings for Part B PAP drugs=sum of (ASP per drug per unit x number of total units } \\
\text { administered to each patient } \times 1.06) \times 80 \% \\
\text { Total reimbursement savings for Part D PAP drugs = sum of (AWP-contract } \% \text { per drug unit x number of total } \\
\text { units administered to each patient) }\end{array}$ \\
\hline $\begin{array}{l}\text { Patient out-of- } \\
\text { pocket savings }\end{array}$ & $\$ 240,350.04$ & Sum of copayments saved from foundation programs (i.e., bona fide independent charities) \\
\hline $\begin{array}{l}\text { Manufacturer } \\
\text { financial impact }\end{array}$ & $\$ 3,379,032.34$ & Sum of AWP of drugs dispensed through PAPs \\
\hline $\begin{array}{l}\text { Health system } \\
\text { missed revenue }\end{array}$ & $\$ 6,481,543.55$ & $\begin{array}{l}\text { Overhead during study period + lost reimbursement } \\
\text { Lost reimbursement }=\text { total missed reimbursement opportunity for Part B PAP medications }+ \text { total missed } \\
\text { reimbursement opportunity for Part D PAP medications } \\
\text { Explanation of reimbursement savings calculations: } \\
\text { Total missed reimbursement opportunity for Part B PAP medications= sum of (ASP per drug per unit } \times \text { number } \\
\text { of total units administered to each patient } \times 1.06 \\
\text { Total missed reimbursement opportunity for Part D PAP medications=sum of (AWP-contract } \% \text { per drug } \\
\text { unit } \times \text { number of total units administered to each patient) }\end{array}$ \\
\hline
\end{tabular}

$P A P=$ patient assistance program; $A S P=$ average sale price; $A W P=$ average wholesale price

technology was used to ensure protection of patient health care information, and data were deidentified for this publication.

The primary outcome of this study was to quantify the overall cost savings to Medicare as a result of patient assistance programs. Secondary outcomes included total Medicare patient out-of-pocket savings as a result of foundation programs, financial effect on manufacturers as a result of patient assistance programs, and total lost institution revenue as a result of assistance efforts. Descriptive statistics were used in analyzing the data. Each outcome was calculated according to Figure 1.

\section{Results}

This study included 114 eligible patients (61 males, 53 females), encompassing a wide variety of malignant hematology and oncology disease states. The average age of study participants was 68.3 years (male average age was 67.9 years; female average age was 68.7 years). Of the total patient population, 108 patients (94.7\%) were Caucasian, and 6 patients (5.3\%) were African American. A wide variety of therapies were prescribed to the study population. The top intravenous therapies included pembrolizumab (Keytruda) and nivolumab (Opdivo). The top oral therapies included capecitabine (Xeloda) and everolimus (Afinitor).

Nine manufacturers provided patient assistance programs: Johnson \& Johnson, Novartis, Pfizer, Astellas Pharma, Merck $\&$ Co., Clovis Oncology, Bristol-Myers Squibb, Amgen, and Janssen. These manufacturers dispensed 2,243 doses through patient assistance programs (1,980 oral doses and 263 injectable doses). Oral doses are typically administered once or twice daily as compared with injectable doses, which may be administered every 2 or 3 weeks. Eight different foundations provided copay assistance for 64 patients in this study: CancerCare, HealthWell Foundation, PAN Foundation, Patient Advocate Foundation, Chronic Disease Fund, Leukemia \& Lymphoma Society, PSI Foundation, and The Assistance Fund.

Patient enrollment in safety net options was supported by 2 full-time equivalent health system employees, resulting in a total labor cost of $\$ 110,000$ annually. Employees at the institution have enrolled patients in safety net options that have contributed significantly to medication financial support (Table 1). Through foundation programs, patients have saved \$240,350.04 in Medicare medication copayments. Manufacturers have contributed by providing $\$ 3,379,032.34$ in free drugs through patient assistance programs.

Health care institutions miss the opportunity for reimbursement when patients use patient assistance programs rather than billing Medicare. For drugs provided by patient assistance programs that would have otherwise been billed under Medicare Part B (e.g., off-label use), the medical center that participated in this study missed an opportunity cost of approximately $\$ 5,888,633.58$ in reimbursements. For drugs provided by patient assistance programs that would have otherwise been billed under Medicare Part D (e.g., off-label use), the medical center missed an opportunity cost of approximately $\$ 372,909.97$. Total lost institutional revenue considering missed reimbursement opportunity and labor expenses was $\$ 6,481,543.55$. Medicare saved $\$ 5,083,816.83$ due to patient assistance programs. 


\section{Discussion}

Medicare saves financially when safety net options are used. When patients enroll in safety net options, especially patient assistance programs, these are savings that are likely not recognized by Medicare because no insurance claim was paid for the medications. Other entities, such as manufacturers and health care institutions, ensure drug access for patients instead. The participating medical center in particular was affected by the missed opportunity for reimbursements under Medicare Part B and Part D because of the use of safety net options. This was all while using the average sale price (ASP) plus $6 \%$ reimbursement rate for Medicare Part B payments to 340B entities. The authors recognize that, in 2018, the reimbursement rate for 340B entities under Medicare Part B was adjusted to ASP minus $22.5 \%$, which would affect current reimbursement calculations. ${ }^{11}$ The original ASP plus $6 \%$ reimbursement rate was used in calculations because of the 2015-2017 time frame of the study (when this reimbursement was standard). Reimbursement calculations for Medicare Part B payments were likely overstated compared with using ASP minus 22.5\% as the reimbursement methodology.

This study also highlights the role that health care institutions, especially academic medical centers, play in assisting patient access to specialty care. When affordability becomes a barrier to appropriate medical therapy, academic medical centers-most markedly those with a strong specialty pharmacy presence-are well equipped to facilitate patient enrollment in financial safety net options. While institutions may be affected financially by this, the focus of these centers on patient care is at the forefront.

An additional element to consider here includes instances where the administration of infusion therapy did not meet Medicare medical necessity coverage. This may be because the therapy was being used outside of 1 of the 6 approved compendia or local coverage determinations used to determine Part B coverage or because of coding errors within the institution's medical record and billing systems. Billing for these services is prohibited, so the resulting claims would be considered bad debt. During the study period, this amount was estimated at $\$ 509,441.29$. Academic medical centers also play a large role in research for a wide variety of diseases, which is another financial effect that may not otherwise be considered and is a driver for many health discoveries in the United States.

From a patient perspective, while foundation programs and patient assistance programs have a substantial effect in gaining access to medication therapy, they may demonstrate benefit in other ways. It is difficult to quantify the value that these foundations and patient assistance programs truly bring. While it is possible to show financial effect, qualitative outcomes, such as readmissions, adherence rates, and quality of life, are often more difficult to estimate.
In the future, research could focus on quantifying the financial effect of patient enrollment in safety net options on other classes of specialty medications. The information presented in this study could also be similarly researched at other health care institutions in order to capture various reimbursement rates, additional manufacturers, and more diverse patient populations. Combining this data would encompass the cumulative financial effect of safety net options for all classes of specialty medications, quantifying the national effect of safety net options on the health care system.

Another interesting approach would be to explore adherence rates and other health outcomes for patients using safety net coverage options compared with those who do not use such services. This approach may further demonstrate the effect of patient assistance and foundation programs on the patient.

\section{Limitations}

This study is not without limitations. Patients were not included who were managed outside the medical center's specialty pharmacy and infusion center or who were lost to follow-up. Patients who participated in institution-sponsored charity programs were also excluded from this study. While this limitation may lessen the financial effect shown in our study because Medicare patients are often included in these programs, not all charity program patients used our pharmacy, which affected the data collection process.

Because Medicare Part D plans are managed by commercial entities, contracts vary by health care entity in regard to reimbursements for oral specialty drugs. This causes variations in financial effect across health care institutions. We also recognize that the cost for a drug manufacturer to manufacture a particular product is likely less than the average wholesale price, which was used because of its public availability.

Finally, the authors acknowledge that drug manufacturers and foundation programs may be linked in some situations, but this idea was not considered when performing analysis for the current study. It was assumed that manufacturers contributed financially to foundation programs, although these entities remain independent as outlined in the Federal Register. ${ }^{12}$

\section{Conclusions}

Patient use of safety net options in obtaining specialty medications results in cost savings to Medicare, as well as to patients. Drug manufacturers are affected financially by providing patient assistance programs to ensure that patients receive access to necessary therapies. Health care institutions miss the opportunity for Medicare reimbursement because of patient enrollment in patient assistance programs and dedicate resources to managing safety net options at the institutional level. Further studies are recommended to quantify the financial effect of safety net options for other disease states, other manufacturers, and other institutions. 


\section{Authors}

STEPHANIE S. BETHAY, PharmD, MBA, BCPS, PGY-2 HealthSystem Pharmacy Administration Resident; STEPHEN K. BATT, PharmD, CSP, Specialty Pharmacy Infusion Services; SAMANTHA H. BOCHENEK, PharmD, MBA, BCPS, Infusion Pharmacy Services; and PHILIP A. SCHWIETERMAN, PharmD, MHA, Markey Cancer Center and Kentucky Children's Hospital, UK HealthCare, Lexington, Kentucky. MATTHEW J. TRAVIS, PharmD, University of Kentucky College of Pharmacy, Lexington.

AUTHOR CORRESPONDENCE: Stephanie S. Bethay, PharmD, MBA, BCPS, UK HealthCare, c/o Philip Schwieterman, 800 Rose St., Rm H110, Lexington, KY 40536. E-mail: stephaniesollis@me.com.

\section{DISCLOSURES}

No outside funding supported this study. The authors have nothing to disclose. Findings from this study were part of a podium research presentation at the Great Lakes Pharmacy Residency Conference; April 25, 2018; West Lafayette, IN

\section{ACKNOWLEDGMENTS}

The authors thank Aaron Cook, PharmD, BCCCP, BCPS, FCCP, FKSHP (UK Health(are) for his assistance throughout this study.

\section{REFERENCES}

1. Centers for Medicare \& Medicaid Services. Announcement of calendar year (CY) 2019 Medicare Advantage capitation rates and Medicare Advantage and Part D payment policies and final call letter. April 2, 2018. Available at: https:/www.cms.gov/Medicare/Health-Plans/MedicareAdvtgSpecRateStats/ Downloads/Announcement2019.pdf. Accessed November 20, 2019.

2. Thomas CP. Key Medicare issues for coverage and reimbursement of specialty pharmaceuticals. The Health Industry Forum. July 2008. Available at: https://healthforum.brandeis.edu/meetings/materials/2008-16-July/ThomasBackground-Paper.pdf. Accessed November 20, 2019.
3. Schwieterman P. Navigating financial assistance options for patients receiving specialty medications. Am J Health Syst Pharm. 2015;72(24):2190-95.

4. Aitken M, Kleinrock M. Medicines use and spending in the U.S.: a review of 2016 and outlook to 2021. IQVIA Institute. May 4, 2017. Available at: https://www.iqvia.com/insights/the-iqvia-institute/reports/medicines-useand-spending-in-the-us-a-review-of-2016. Accessed December 6, 2019.

5. Aitken M, Kleinrock M. Medicine use and spending in the U.S.: a review of 2017 and outlook to 2022. IQVIA Institute for Human Data Science. April 19, 2018. Available at: https://www.iqvia.com/institute/reports/medicine-use-and-spending-in-the-us-review-of-2017-outlook-to-2022. Accessed November 20, 2019.

6. Martin AB, Hartman M, Washington B, Catlin A. National health care spending in 2017: growth slows to post-Great Recession rates; share of GDP stabilizes. Health Aff (Millwood). 2019;38(1):96-106.

7. Dusetzina SB, Keating NL. Mind the gap: why closing the doughnut hole is insufficient for increasing Medicare beneficiary access to oral chemotherapy. J Clin Oncol. 2016;34(4):375-81.

8. Rosenthal T. High costs lead cancer patients to abandon or delay therapy. Specialty Pharmacy Continuum. August 2, 2018. Available at: https://www. specialtypharmacycontinuum.com/Clinical/Article/08-18/High-Costs-LeadCancer-Patients-to-Abandon-or-Delay-Therapy/52366. Accessed November 20, 2019.

9. Mitchell A, Muluneh B, Patel R, Basch E. Pharmaceutical assistance programs for cancer patients in the era of orally administered chemotherapeutics. J Oncol Pharm Practice. 2018;24(6):424-32.

10. Khan G, Karabon P, Lerchenfeldt S. Use of prescription assistance programs after the Affordable Care Act. J Manag Care Spec Pharm. 2018;24(3):247-51. Available at: https://www.jmcp.org/doi/10.18553/ jmcp. 2018.24.3.247.

11. Centers for Medicare \& Medicaid Services. Medicare-FFS Program. Billing 340B modifiers under the Hospital Outpatient Prospective Payment System (OPPS). April 2, 2018. Available at: https://www.cms.gov/medicare/ medicare-fee-for-service-payment/hospitaloutpatientpps/downloads/billing340b-modifiers-under-hospital-opps.pdf. Accessed November 20, 2019.

12. Levinson DR. Publication of OIG special advisory bulletin on patient assistance programs for Medicare Part D enrollees. Fed Reg. 2005;70(224):70623-70628. Available at: https://www.govinfo.gov/content/ pkg/FR-2005-11-22/html/05-23038.htm. Accessed November 20, 2019. 